\title{
LA REFORMA DEL PROGRAMA DE FORMACIÓN GENERAL EN LA PONTIFICIA UNIVERSIDAD CATÓLICA DE CHILE: BASES GENERALES
}

Nicolás Velasco* 


\section{RESUMEN}

Para dar fiel cumplimiento a sus objetivos educacionales declarados, la Pontificia Universidad Católica de Chile reformó sus programas de formación general. El programa actual es de 80 créditos, de los cuales 20 corresponden a cursos del área de Teología, Antropología y Ética y los 60 restantes a cursos regulares de carreras y licenciaturas de disciplinas diferentes a las incluidas en el currículo de la carrera que cursa el alumno, a quien se le exigen además requisitos de competencia en castellano e inglés.

Se pretende que este programa permita a los alumnos un aprendizaje extendido, desarrollando habilidades en comunicación y para trabajar en equipo, además de respeto y tolerancia por personas diferentes a las de su profesión.

Nuestro plan de desarrollo contempla una serie de acciones para fortalecer el currículo implícito, las que facilitan el estudio de los alumnos, el desarrollo de capacidades de aprendizaje autónomo y el sentido solidario.

\section{ABSTRACT}

In order to comply with its stated educational goals, the Pontificia Universidad Católica de Chile has modified its general education program. The current program requires 80 credits, divided as follows: 20 credits in the area of Theology, Anthropology and Ethics, with the remaining 60 credits assigned to regular courses in the different programs and licentiate-track courses followed by the students depending on the syllabus they have chosen. In addition, students are required to comply with some linguistic skills in Spanish and English.

The underlying idea in this new program, is to allow the students to have an extended learning experience, to have them develop permanent skills in communication, to make them competent in team work.

Furthermore, the development plan proposed by the Pontificia Universidad Católica de Chile involves a series of actions intended to strengthen the implicit curriculum. All of these are factors that make studying easier for students and help them to develop independent learning skills and a sense of solidarity. 


\section{LA REFORMA DEL PROGRAMA DE FORMACIÓN GENERAL EN LA PONTIFICIA UNIVERSIDAD CATÓLICA DE CHILE: BASES GENERALES}

\section{INTRODUCCIÓN}

El objetivo del presente artículo es esbozar las bases conceptuales de la modificación de nuestro proyecto educativo y mostrar su coherencia con los principios de la Iglesia y la Universidad y su concordancia con las tendencias vigentes para la educación superior.

Nuestra Universidad, en su declaración de principios de 1979, artículo 8, establece:

"La Universidad aspira a lograr una educación sólida, arraigada en la ciencia, el arte y la moral, penetrada por el espíritu que anima a esta casa de estudios, por el amor a la cultura y por el servicio a los hombres en quienes se sirve a Dios. Desea, por lo tanto, que todos los que estudian en ella no resulten sólo científica y técnicamente capacitados, sino que estén abiertos a las distintas dimensiones de lo humano, conscientes de su responsabilidad personal y social e impregnados de un espíritu cristiano que los capacite para asumir en forma efectiva una acción orientadora y conductora frente a los desafíos que plantea el desarrollo integral de una sociedad libre de las injusticias que el pecado y los errores de los hombres han permitido o establecido y que afectan a vastos sectores que sufren sus consecuencias espirituales y materiales" ${ }^{1}$.

Esta propuesta resulta iluminadora con respecto a las tareas docentes que son específicas de una Universidad católica y pioneras de las actuales tendencias educativas para la educación superior. En efecto, su espíritu y letra concuerdan con el Magisterio de la Iglesia en cuanto a educación universitaria y abordan muchas de las características

1. Declaración de Principios, Pontificia Universidad Católica de Chile, 1979. 
del proceso educativo, que diferentes organismos internacionales han recomendado desarrollar en aquellas universidades que pretenden formar líderes y servir eficientemente al progreso de la sociedad.

Desde hace mucho tiempo, en períodos anteriores y posteriores a esta declaración de principios, nuestra Universidad ha desarrollado varias iniciativas para ser fiel a la tarea educativa que es propia de su misión. Dichos esfuerzos se reflejan en los currículos de varias carreras y en los cursos o programas específicos, dirigidos a ofrecer a los alumnos una educación más amplia que la mera formación profesional. Los programas de formación general se establecieron con el fin declarado de formar personas cultas, capaces de "conocer, comprender, interpretar y utilizar, a lo menos en un primer nivel, los distintos tipos de lenguaje con los que estructuramos e interpretamos la realidad: el científico, el artístico, el filosófico y el cinético; capaces de vivir de acuerdo con las ideas de su tiempo y de renovar las que deben ser cambiadas; capaces de integrar su actuación y su cultura dentro de los principios de la ética y la teología, obteniendo así la libertad ética base de la libertad psicológica; capaces de interpretar la realidad desde una perspectiva histórica, entendiendo así las distintas respuestas que se dan a los problemas del mundo y del hombre"2.

Como se puede apreciar, desde hace mucho tiempo se cuenta con una visión muy completa de lo que deben ser las características de nuestra educación. Sin embargo, a pesar de lo anterior, los programas de formación general destinados a cumplir estos objetivos se han aplicado de manera restringida en extensión y mediante cursos de calidad variable. Por lo anterior, su eficacia para alcanzar los objetivos educacionales planteados ha sido menor. Por otra parte, no ha existido una política sostenida en el largo plazo, para la aplicación o evaluación de los diferentes planes y programas.

A partir de 1993, la Universidad establece el Programa de Bachillerato, con un componente mayor de flexibilidad y de formación multidisciplinaria. Dicho programa, pionero en nuestro medio, es un aporte importante para la Universidad y para el medio universitario chileno: constituye una manera de poner en práctica los altos objetivos

2. Política Académica de Formación General, Pontificia Universidad Católica de Chile, 1978. 
declarados en 1978 y 1979; le ha proporcionado a la Universidad una importante cantidad de alumnos con altos puntajes en la PAA; ha validado un grado académico precoz dentro de los estudios universitarios y, de este modo, ha colaborado a la introducción de una mayor flexibilidad y articulación en la enseñanza universitaria.

A partir del año 2000, nuestra comunidad académica es convocada a elaborar un plan de desarrollo para el período 2000-2005. A fines del mismo año, el Honorable Consejo Superior aprueba por unanimidad el documento final de dicho plan. Este documento es un nuevo y significativo paso en pos del logro de los objetivos escritos en nuestra declaración de principios. En primer término, aborda la definición explícita de nuestros objetivos educacionales comunes, los que, junto a la excelencia en la formación profesional o disciplinaria, proporcionarán nuestro sello distintivo a los egresados y, por otra parte, caracteriza el detalle de dichos objetivos. Además, este plan reconoce que la tarea educativa es responsabilidad primera de los profesores y de toda la comunidad universitaria y que, por lo tanto, el cumplimiento de los objetivos educacionales comunes no es sólo responsabilidad de un conjunto de cursos sino, más bien, de dichos cursos y del currículo implícito que los sustenta. Desde el punto de vista anterior, se hace necesario mirar todas las acciones del plan de desarrollo como relacionadas entre sí, para proporcionar al Programa de Formación General la fuerza de docencia y testimonio que éste requiere para ser eficaz. Algunos de los componentes de este currículo implícito son: la selección y capacitación de profesores, el cambio de los paradigmas en métodos docentes, el incremento en disponibilidad de medios computacionales para la enseñanza, el impulso para el desarrollo de una actividad pastoral y solidaria amplia, el estímulo para el intercambio internacional y la promoción de la interdisciplina.

\section{BASES CONCEPTUALES DE LA FORMACIÓN GENERAL}

El Magisterio de SS Juan Pablo II ha sido muy claro en definir las características centrales de un proyecto universitario católico. Nuestro plan de desarrollo acoge dichas enseñanzas y las renovaciones que de ella derivan. Como consecuencia de lo anterior, el proyecto educativo 
debe orientarse "hacia la formación de personas cultas, capaces de desarrollar visiones unitarias del fragmentado saber contemporáneo y también competentes, emprendedoras y solidarias, animadas por valores cristianos" ${ }^{3}$. Lo anterior es fiel a lo declarado por el Santo Padre: "La sociedad no sólo pide a la Universidad especialistas, cerrados en sus campos específicos del saber, de la cultura, de la ciencia y de la técnica, pero, por sobre todo, constructores de humanidad, servidores de la comunidad de hermanos, promotores de la justicia por su orientación a la verdad. En una palabra, hoy, como siempre, son necesarias personas de cultura y de ciencia, que sepan poner los valores de la conciencia sobre el resto, y cultivar la supremacía del ser sobre el parecer"4.

Crear un proyecto educativo con las características descritas por el Santo Padre requiere la conjunción de varios aspectos. El primero y más importante es la presencia de profesores capaces de transmitir la pasión por el "descubrimiento" de lo enseñado y de construir una macrovisión a partir de un detalle de su materia y, de ese modo, hacerlo partícipe de valores universales.

Quien haya tenido maestros con las capacidades previamente descritas, recuerda en ellos dos características comunes: integridad y entusiasmo. Una tercera condición ayuda al logro de esta maestría: cultura. Cultura entendida como "no sólo de las cosas en sí, sino en todo aquello que está entre las cosas. Es decir, el mundo de las relaciones. Toda realidad es un nudo, una urdiembre, un entrecruzamiento de relaciones"5.

Por lo tanto, el profesor por sí mismo y a su vez el programa de estudios, debieran acrecentar su capacidad de integrar al alumno en una visión del mundo como relación, es decir "que cualquiera sea su opción disciplinaria, esté apto para percatarse de que toda realidad es susceptible de ser interpretada como mero objeto aislado o como inserta en un ámbito. La conciencia del ámbito es la que crea el imperativo de la relación"6. Esta misma conciencia de ámbito, lograda a través del

3. Plan de Desarrollo 2000-2005, Pontificia Universidad Católica de Chile.

4. SS Juan Pablo II, Discurso de Turín, Italia, 1988.

5. Jornadas de Docencia, Pontificia Universidad Católica de Chile, 2001.

6. Ibídem. 
conocimiento de otras disciplinas y de quienes las practican, genera un incremento en la tolerancia y una disminución en "los prejuicios, el dogmatismo y el autoritarismo"'.

La educación, entendida como el sacar a luz de un modo perfectivo las potencialidades del ser, exige conocerse a sí mismo. Tal como señala SS Juan Pablo II:

"Conócete a ti mismo... debe ser asumida como la regla mínima para todo hombre deseoso de distinguirse en medio de toda la creación, calificándose como hombre precisamente como conocedor de sí mismo" . Nuestra Universidad debe permitir y, más aún, promover el conocimiento de sí mismo para desarrollar una real humanización del hombre y, por medio de su acción, de la cultura misma. Tal como lo expresara nuestro Arzobispo fundador, Don Mariano Casanova, la Pontificia Universidad Católica de Chile está llamada a "colaborar en la misión de la Iglesia de llevar al hombre a la plenitud de la edad, a la medida del hombre perfecto" 9 .

La cultura universitaria puede posibilitar u obstaculizar la humanización. Para posibilitarla, debe colaborar al tener que deriva de la praxis, pero también al ser de los estudiantes y de toda la comunidad universitaria. Lo anterior no significa una antinomia entre la enseñanza de la praxis y el desarrollo del ser. Por el contrario, dicho proceso educativo puede proveer de una formación que, además de entregar un medio para insertarse en la sociedad, permita desarrollar una serie de habilidades permanentes que favorecen el logro de la anhelada humanización. Sin embargo, la excesiva concentración curricular en materias propias del oficio disminuye la armonía en la formación de los estudiantes, reduce su posibilidad de apreciar distintos ámbitos del saber y, por lo tanto, reduce sus posibilidades de percibir el mundo como relaciones, limitando su capacidad de lograr una cultura verdaderamente humana. Por último, el conocerse a sí mismo se favorece por medio de una formación universitaria que facilita el conocer lo que otros son y cómo construyen su saber.

7. Derek, Bok, Higher Learning, Harvard University Press, Cambridge, EE.UU., 1986.

8. Encíclica Fides et Ratio, Ediciones Paulinas, Santiago, Chile, 1998, N 1.

9. Rosso, Pedro, discurso con motivo del día de la Universidad: "Educación y cultura: una paideia para los nuevos tiempos", Santiago, Chile, junio, 2000. 
Sin embargo, y a pesar de que la organización curricular puede proveer el humus necesario para el logro de una cultura humana, debe reconocerse que el currículo es sólo un instrumento en pos de ese alto objetivo. Lo fundamental para lograr una verdadera educación integral es una comunidad de profesores y alumnos que se propongan profundizar en el conocimiento de sí mismos. Este es el punto de origen a partir del cual los hombres se interrogan acerca del sentido de su vida y de su oficio. Este es un objetivo central que debe perseguir la educación universitaria católica. Es por ello que nuestro desarrollo educacional no depende sólo de un currículo; es por esto que nos hemos propuesto un plan de desarrollo global, para ser terreno fértil de una educación que promueva la humanización.

En las palabras del Padre Giussani, se ilumina el horizonte de la Iglesia con respecto a la actitud básica de la educación: "La primera preocupación de una educación verdadera y adecuada es la de educar el corazón del hombre así como Dios lo ha hecho. La moral no es otra cosa que continuar la actitud original con la cual Dios crea al hombre frente a todas las cosas y en su relación con ellas". Estas reflexiones nos dan un punto de origen y cimiento a toda nuestra actividad universitaria, basada en una inteligencia que participa en "la búsqueda de la verdad y la certeza de conocer ya la fuente de la verdad"10.

\section{EL PROGRAMA DE FORMACIÓN GENERAL: ANTECEDENTES GENERALES}

El logro de los objetivos educacionales comunes que nos hemos propuesto debe sustentarse en la comunidad universitaria y en acciones curriculares eficaces. Dos de las características que favorecen la efectividad de dichos currículos son su extensión y su rigurosidad.

Resulta evidente que los objetivos de 1978, 1979 o del 2000 son muy difíciles de lograr con un Programa de Formación General como el vigente hasta el año 2001, de sólo 20 créditos. Nuestra cultura curricular reconoce que esta extensión es menor. Si analizamos con

10. Constitución Apostólica del Sumo Pontífice Juan Pablo II sobre las Universidades Católicas, Ex Corde Ecclesiae, Ediciones Paulinas, Santiago, Chile, 1990, N 1. 
detención los diversos currículos de las licenciaturas vigentes, 20 créditos es la extensión usada para dar cierta base de formación en: análisis cuantitativo para un periodista, la mitad de la enseñanza en matemáticas para un químico farmacéutico, las bases en química para una enfermera, la enseñanza en economía para un trabajador social, la extensión de sólo un curso de fisiología para medicina y la cuarta parte de la formación en matemáticas de un ingeniero.

Antes de analizar la extensión que el programa debiera tener, es necesario reflexionar acerca de los cursos que deben constituir dicho programa. Si el objetivo es ser riguroso para integrar a los estudiantes en forma real, dentro del mundo de otras disciplinas, las asignaturas debieran tener una extensión suficiente para garantizar, de esta manera, una inmersión disciplinaria eficaz. Esos cursos debieran ser dictados por profesores que cultivan la materia que enseñan. Este tipo de cursos son, en general, los denominados mínimos y los OPR de las licenciaturas (en general de 10 créditos). El tomar esta clase de cursos tiene ventajas asociadas. En primer término, ellos son impartidos por profesores exigentes y con trayectoria, y son los más "cercanos" y mejor cuidados por la unidad que los dicta. Por otra parte, al tomar esta clase de cursos, el estudiante tendrá un importante contacto con profesores y alumnos de carreras diferentes a la propia, lo que lo familiarizará más profundamente con las maneras de conocer que otros tienen. Los estudios de impacto del College de Harvard demuestran que los estudiantes obtienen eficientemente las competencias deseadas cuando efectúan cursos con las características descritas previamente. Por el contrario, aquellos que toman sólo cursos de corte general o descriptivo, "vuelven atrás en sus grados de competencia, a niveles similares a los que tenían antes de ingresar al college"11. El mismo estudio demuestra que los estudiantes que viven en el campus, comparados con aquellos que residen fuera de él, logran mejores capacidades de tolerancia por otros, así como de autoconocimiento y de relaciones personales. Lograr que los alumnos permanezcan un mayor tiempo en la Universidad y que convivan académicamente con estudiantes de otras disciplinas, puede ayudar a obtener resultados similares. 
Si deseamos que nuestros egresados sean promotores de cambios positivos en el país, debemos entregarles formación y herramientas para tener éxito en su tarea. Es nuestra obligación proveerlos de sentido moral, de respeto y acogida a los que no son como él y de una cultura básica que les permita insertarse en el mundo, abordar los complejos problemas que aquejan a nuestra sociedad y liderar equipos de trabajo. Para los fines anteriores, se ha diseñado un programa complementario a las licenciaturas, cuyos objetivos se describen a continuación.

Un primer objetivo para este Programa de Formación General, particular y distintivo de nuestro proyecto educativo, es el mandato de integrar la reflexión acerca de la teología y de lo antropológicoético dentro del currículo de todas las licenciaturas. Para servir adecuadamente este objetivo, se considera que los 20 créditos que actualmente constituyen el requisito mínimo para la formación general, deben ser destinados a cubrir sólo estas áreas.

El segundo objetivo es lograr que los alumnos se familiaricen con disciplinas distintas a las centrales del oficio que aprenden. Para lograr este propósito, la extensión mínima que debería tener el programa es la equivalente a un certificado académico (conjunto de cinco cursos acerca de una disciplina o de un tema, equivalente a un semestre de estudios o 50 créditos). Esta extensión permite al estudiante explorar con eficacia otro campo del saber y, en el caso de que elija cursos individuales en varias disciplinas, le da la oportunidad de cubrir con cierta solvencia todos los objetivos de formación general planteados en el plan de desarrollo 2000-2005.

Somos conscientes de que permitir a los estudiantes cumplir con su formación general en forma de un certificado académico, puede inducirlos a no obtener todos los objetivos programados. Sin embargo, la profundización disciplinaria, propia de estos certificados, proporciona logros asociados en la "apreciación del ámbito" y, en consecuencia, una deseable solvencia en lo aprendido.

Para este segundo objetivo, se plantea una extensión de 60 créditos, suficiente para asegurar que todos los alumnos, aunque cursen un certificado académico, tomen cursos por lo menos en dos áreasobjetivo de formación general. 
Con el fin de especificar las disciplinas en que los alumnos podrán tomar sus cursos, se tendrá en consideración la estructura de la propia licenciatura, teniendo presente que, tal como se planteó en el plan de desarrollo, el Programa de Formación General es complementario de ella. Se considera que esta licenciatura es lo central de los estudios universitarios, ya que refleja el desarrollo en profundidad de un área del saber, capacita para la praxis y refleja lo esencial de la actividad universitaria: "Esta relación creativa con la verdad en un sector determinado del conocimiento y de la ciencia constituye la substancia propia de los estudios a nivel universitario"12. Por otra parte, este estudio en profundidad en un área del saber también proporciona ciertas habilidades permanentes y algunos conocimientos compatibles con nuestros objetivos educacionales comunes.

Un tercer objetivo es proporcionar ciertas competencias lingüísticas imprescindibles para todo profesional. Éstas son la capacidad de comprensión de textos, de redactar en forma correcta en castellano y el dominio del inglés en un nivel que permita comprender textos escritos y acceder a Internet en forma eficaz. Lo anterior, además de constituir una herramienta clave para su trabajo académico, le será de gran utilidad en el ejercicio de su carrera o disciplina.

El dominio básico del inglés permite consultar una gran cantidad de literatura y bases de datos que, de otra manera, serían incomprensibles. Además, el mundo global usa el inglés como lingua franca, por lo que el dominio de este idioma también es parte integral del ejercicio de muchas profesiones y, además, una herramienta eficiente para "aprender a aprender".

El logro de estos objetivos se medirá a través de exámenes de competencias y, los alumnos que reprueben, serán apoyados por cursos presenciales y herramientas docentes en la web.

La enseñanza y medición de la capacidad de escritura en castellano estará integrada en al menos tres cursos mínimos de cada licenciatura, a través de la corrección rigurosa de artículos elaborados por los estudiantes. Los aspectos evaluados serán los propiamente disciplinarios y aquellos referidos a redacción y expresión de ideas.

12. SS Juan Pablo II, Carta a los Universitarios de México y de América Latina. 
Todas éstas son las bases conceptuales que han dado origen al nuevo plan de formación aprobado por el Honorable Consejo Superior. Todas las licenciaturas de la Universidad se integrarán a él a partir del año 2003. Con esto se cumple uno de los objetivos centrales del Plan de Desarrollo 2000-2005. 